\title{
The distance between a naive cumulative estimator and its least concave majorant
}

\author{
Hendrik P. Lopuhaäa, Eni Musta ${ }^{\mathrm{a}, *}$ \\ ${ }^{a}$ DIAM, Faculty EEMCS, Delft University of Technology, Mekelweg 4, 2628 CD Delft, The Netherlands
}

\begin{abstract}
We consider the process $\widehat{\Lambda}_{n}-\Lambda_{n}$, where $\Lambda_{n}$ is a cadlag step estimator for the primitive $\Lambda$ of a nonincreasing function $\lambda$ on $[0,1]$, and $\widehat{\Lambda}_{n}$ is the least concave majorant of $\Lambda_{n}$. We extend the results in Kulikov and Lopuhaä (2006, 2008) to the general setting considered in Durot (2007). Under this setting we prove that a suitably scaled version of $\widehat{\Lambda}_{n}-\Lambda_{n}$ converges in distribution to the corresponding process for two-sided Brownian motion with parabolic drift and we establish a central limit theorem for the $L_{p}$-distance between $\widehat{\Lambda}_{n}$ and $\Lambda_{n}$.

Keywords: Least concave majorant, Grenander-type estimator, Limit distribution, Central limit theorem for $L_{p}$-distance, Brownian motion with parabolic drift

2010 MSC: 60F05, 62E20
\end{abstract}

\section{Introduction}

Grenander-type estimators are well known methods for estimation of monotone curves. In case of estimating nonincreasing curves, they are constructed by starting with a naive estimator for the primitive of the curve of interest and then take the left-derivative of the least concave majorant (LCM) of the naive estimator. The first example can be found in Grenander (1956) in the context of estimating a nonincreasing density $f$ on $[0, \infty)$ on the basis of an i.i.d. sample from $f$. The empirical distribution function $F_{n}$ of the sample is taken as a naive estimator for the cumulative distribution function corresponding to $f$ and the Grenander estimator is found by taking the leftderivative $\widehat{f}_{n}$ of the least concave majorant $\widehat{F}_{n}$. Similar estimators have been developed in other statistical models, e.g., regression (see Brunk (1958)), random censoring (see Huang and Wellner (1995)), or the Cox model (see Lopuhä̈ and Nane (2013)). Durot (2007) considers Grenandertype estimators in a general setup that incorporates several statistical models. A large part of the literature is devoted to investigating properties of Grenander-type estimators for monotone curves, and somewhat less attention is paid to properties of the difference between the corresponding naive estimator for the primitive of the curve and its LCM.

\footnotetext{
${ }^{*}$ Corresponding author

Email address: e.musta@tudelft.nl (Eni Musta)
} 
Kiefer and Wolfowitz (1976) show that $\sup _{t}\left|\widehat{F}_{n}-F_{n}\right|=O_{p}\left(\left(n^{-1} \log n\right)^{2 / 3}\right)$. Although the first motivation for this type of result has been asymptotic optimality of shape constrained estimators, it has several important statistical applications. The Kiefer-Wolfowitz result was a key argument in Sen et al. (2010) to prove that the $m$ out of $n$ bootstrap from $\widehat{F}_{n}$ works. Mammen (1991) suggested to use the result to make an asymptotic comparison between a smoothed Grenander-type estimator and an isotonized kernel estimator in the regression context. See also Wang and Woodroofe (2007) for a similar application of their Kiefer-Wolfowitz comparison theorem. An extension to a more general setting was established in Durot and Lopuhaä (2014), which has direct applications in Durot et al. (2013) to prove that a smoothed bootstrap from a Grenander-type estimator works for $k$-sample tests, and in Groeneboom and Jongbloed (2013) and Lopuhaä and Musta (2017) to extract the pointwise limit behavior of smoothed Grenander-type estimators for a monotone hazard from that of ordinary kernel estimators. To approximate the $L_{p}$-error of smoothed Grenander-type estimators by that of ordinary kernel estimators, such as in Csörgö and Horváth (1988) for kernel density estimators, a Kiefer-Wolfowitz type result no longer suffices. In that case, results on the $L_{p}$-distance, between $\widehat{F}_{n}$ and $F_{n}$ are more appropriate, such as the ones in Durot and Tocquet (2003) and Kulikov and Lopuhaä (2008).

In this paper, we extend the results in Durot and Tocquet (2003) and Kulikov and Lopuhaä (2008) to the general setting of Durot (2007). Our main result is a central limit theorem for the $L_{p}$-distance between $\widehat{\Lambda}_{n}$ and $\Lambda_{n}$, where $\Lambda_{n}$ is a naive estimator for the primitive $\Lambda$ of a monotone curve $\lambda$ and $\widehat{\Lambda}_{n}$ is the LCM of $\Lambda_{n}$. As special cases we recover Theorem 5.2 in Durot and Tocquet (2003) and Theorem 2.1 in Kulikov and Lopuhaä (2008). Our approach requires another preliminary result, which might be of interest in itself, i.e., a limit process for a suitably scaled difference between $\widehat{\Lambda}_{n}$ and $\Lambda_{n}$. As special cases we recover Theorem 1 in Wang (1994), Theorem 4.1 in Durot and Tocquet (2003), and Theorem 1.1 in Kulikov and Lopuhaä (2006).

\section{Main results}

We consider the general setting in Durot (2007). Let $\lambda:[0,1] \rightarrow \mathbb{R}$ be nonincreasing and assume that we have at hand a cadlag step estimator $\Lambda_{n}$ of

$$
\Lambda(t)=\int_{0}^{t} \lambda(u) \mathrm{d} u, \quad t \in[0,1] .
$$

In the sequel we will make use of the following assumptions.

(A1) $\lambda$ is strictly decreasing and twice continuously differentiable on $[0,1]$ with $\inf _{t}\left|\lambda^{\prime}(t)\right|>0$.

(A2) Let $B_{n}$ be either a Brownian motion or a Brownian bridge. There exists $q>6, C_{q}>0$, $L:[0,1] \rightarrow \mathbb{R}$, and versions of $M_{n}=\Lambda_{n}-\Lambda$ and $B_{n}$ such that

$$
\mathbb{P}\left(n^{1-1 / q} \sup _{t \in[0,1]}\left|M_{n}(t)-n^{-1 / 2} B_{n} \circ L(t)\right|>x\right) \leq C_{q} x^{-q}
$$


for all $x \in(0, n]$. Moreover, $L$ is increasing and twice differentiable on $[0,1]$, with $\sup _{t}\left|L^{\prime \prime}(t)\right|<$ $\infty$ and $\inf _{t}\left|L^{\prime}(t)\right|>0$.

Note that this setup includes several statistical models, such as monotone density, monotone regression, and the monotone hazard model under random censoring, see Durot (2007) [Section 3].

We consider the distance between $\Lambda_{n}$ and its least concave majorant $\widehat{\Lambda}_{n}=\mathrm{CM}_{[0,1]} \Lambda_{n}$, where $\mathrm{CM}_{I}$ maps a function $h: \mathbb{R} \rightarrow \mathbb{R}$ into the least concave majorant of $h$ on the interval $I \subset \mathbb{R}$. Consider the process

$$
A_{n}(t)=n^{2 / 3}\left(\widehat{\Lambda}_{n}(t)-\Lambda_{n}(t)\right), \quad t \in[0,1]
$$

and define

$$
Z(t)=W(t)-t^{2}, \quad \zeta(t)=\left[\mathrm{CM}_{\mathbb{R}} Z\right](t)-Z(t),
$$

where $W$ denotes a standard two-sided Brownian motion originating from zero. For each $t \in(0,1)$ fixed and $t+c_{2}(t) s n^{-1 / 3} \in(0,1)$, define

$$
\zeta_{n t}(s)=c_{1}(t) A_{n}\left(t+c_{2}(t) s n^{-1 / 3}\right)
$$

where

$$
c_{1}(t)=\left(\frac{\left|\lambda^{\prime}(t)\right|}{2 L^{\prime}(t)^{2}}\right)^{1 / 3}, \quad c_{2}(t)=\left(\frac{4 L^{\prime}(t)}{\left|\lambda^{\prime}(t)\right|^{2}}\right)^{1 / 3} .
$$

Our first result is the following theorem, which extends Theorem 1.1 in Kulikov and Lopuhaä (2006).

Theorem 1. Suppose that assumptions (A1)-(A2) are satisfied. Let $\zeta_{n t}$ and $\zeta$ be defined in (3) and (2). Then the process $\left\{\zeta_{n t}(s): s \in \mathbb{R}\right\}$ converges in distribution to the process $\{\zeta(s): s \in \mathbb{R}\}$ in $D(\mathbb{R})$, the space of cadlag function on $\mathbb{R}$.

Note that as a particular case $\zeta_{n t}(0)$ converges weakly to $\zeta(0)$. In this way, we recover Theorem 1 in Wang (1994) and Theorem 4.1 in Durot and Tocquet (2003). The proof of Theorem 1 follows the line of reasoning in Kulikov and Lopuhaä (2006).

Let us briefly sketch the argument to prove Theorem 1 Note that $A_{n}=D_{[0,1]}\left[n^{2 / 3} \Lambda_{n}\right]$ and $\zeta=D_{\mathbb{R}}[Z]$, where $D_{I} h=\mathrm{CM}_{I} h-h$, for $h: \mathbb{R} \rightarrow \mathbb{R}$. Since $D_{I}$ is a continuous mapping, the main idea is to apply the continuous mapping theorem to properly scaled approximations of the processes $\Lambda_{n}$ and $Z$ on a suitable chosen fixed interval $I$. The first step is to determine the weak limit of $\Lambda_{n}$, which is given in the following lemma.

Lemma 2. Suppose that assumptions (A1)-(A2) are satisfied. Then for $t \in(0,1)$ fixed, the process $X_{n t}(s)=n^{2 / 3}\left(\Lambda_{n}\left(t+s n^{-1 / 3}\right)-\Lambda_{n}(t)-\left(\Lambda\left(t+s n^{-1 / 3}\right)-\Lambda(t)\right)\right)$ converges in distribution to the process $\left\{W\left(L^{\prime}(t) s\right): s \in \mathbb{R}\right\}$. 
Since $n^{2 / 3}\left(\Lambda\left(t+s n^{-1 / 3}\right)-\Lambda(t)\right) \approx n^{1 / 3} \lambda(t) s+\lambda^{\prime}(t) s^{2} / 2$ and $D_{I}$ is invariant under addition of linear functions, it follows that the process $A_{n}$ can be approximated by a Brownian motion with a parabolic drift. The idea now is to use continuity of $D_{I}$, for a suitably chosen interval $I=[-d, d]$, to show that $D_{I} E_{n t}$ converges to $D_{I} Z_{t}$, where

$$
\begin{aligned}
E_{n t}(s) & =n^{2 / 3} \Lambda_{n}\left(t+s n^{-1 / 3}\right) \\
Z_{t}(s) & =W\left(L^{\prime}(t) s\right)+\lambda^{\prime}(t) s^{2} / 2 .
\end{aligned}
$$

In order to relate this to the processes $\zeta_{n t}$ and $\zeta$ in Theorem 1, note that $A_{n}\left(t+s n^{-1 / 3}\right)=$ $\left[D_{I_{n t}} E_{n t}\right](s)$, where $I_{n t}=\left[-t n^{1 / 3},(1-t) n^{1 / 3}\right]$, and by Brownian scaling, the process $Z(s)$ has the same distribution as the process $c_{1}(t) Z_{t}\left(c_{2}(t) s\right)$. This means that we must compare the concave majorants of $E_{n t}$ on the intervals $I_{n t}$ and $I$, as well as the concave majorants of $Z_{t}$ on the interval $I$ and $\mathbb{R}$. Lemma 1.2 in Kulikov and Lopuhaä (2006) shows that, locally, with high probability, both concave majorants of the process $Z_{t}$ coincide on $[-d / 2, d / 2]$, for large $d>0$. A similar result is established for the concave majorants of the process $E_{n t}$ in Lemma 3, which is analogous to Lemma 1.3 in Kulikov and Lopuhaä (2006). As a preparation for Theorem 4, the lemma also contains a similar result for a Brownian motion version of $E_{n t}$.

Let $B_{n}$ be as in assumption (A2) and let $\xi_{n}$ be a $N(0,1)$ distributed random variable independent of $B_{n}$, if $B_{n}$ is a Brownian bridge, and $\xi_{n}=0$, when $B_{n}$ is a Brownian motion. Define versions $W_{n}$ of a Brownian motion by $W_{n}(t)=B_{n}(t)+\xi_{n} t$, for $t \in[0,1]$, and define

$$
A_{n}^{W}=n^{2 / 3}\left(\mathrm{CM}_{[0,1]} \Lambda_{n}^{W}-\Lambda_{n}^{W}\right)
$$

where $\Lambda_{n}^{W}(t)=\Lambda(t)+n^{-1 / 2} W_{n}(L(t))$, with $L$ as in assumption (A2). Furthermore, define $E_{n}=$ $\sqrt{n}\left(\Lambda_{n}-\Lambda\right), \Lambda_{n}^{E}=\Lambda_{n}, A_{n}^{E}=A_{n}$. The superscripts $E$ and $W$ refer to the empirical and Brownian motion version. For $d>0$, let $I_{n t}(d)=[0,1] \cap\left[t-d n^{-1 / 3}, t+d n^{-1 / 3}\right]$ and, for $J=E, W$, define the event

$$
N_{n t}^{J}(d)=\left\{\left[\mathrm{CM}_{[0,1]} \Lambda_{n}^{J}\right](s)=\left[\mathrm{CM}_{I_{n t}(d)} \Lambda_{n}^{J}\right](s), \text { for all } s \in I_{n t}(d / 2)\right\} .
$$

Let $I_{n t}=I_{n t}(\log n)$ and $N_{n t}^{J}=N_{n t}^{J}(\log n)$.

Lemma 3. Assume that assumptions (A1)-(A2) hold. For $d>0$, let $N_{n t}^{J}(d)$ be the event defined in (7). There exists $C>0$, independent of $n, t, d$, such that

$$
\begin{aligned}
& \mathbb{P}\left(\left(N_{n t}^{W}(d)\right)^{c}\right)=O\left(\mathrm{e}^{-C d^{3}}\right) \\
& \mathbb{P}\left(\left(N_{n t}^{E}(d)\right)^{c}\right)=O\left(n^{1-q / 3} d^{-2 q}+\mathrm{e}^{-C d^{3}}\right),
\end{aligned}
$$

where $q$ is from assumption (A2).

The proof of Theorem 1 now follows the same line of reasoning as that of Theorem 1.1 in Kulikov and Lopuhaä (2006), see Section 3 for more details. The next step is to deal with the $L_{p}$ norm. Our main result is the following. 
Theorem 4. Suppose that assumptions (A1)-(A2) are satisfied and let $A_{n}$ and $\zeta$ be defined by (10) and (2), respectively. Let $\mu$ be a measure on the Borel sets of $\mathbb{R}$, such that

(A3) $\mathrm{d} \mu(t)=w(t) \mathrm{d} t$, where $w(t) \geq 0$ is differentiable with bounded derivative on $[0,1]$.

Then, for all $1 \leq p<\min (q, 2 q-7)$, (with $q$ as in assumption (A2)),

$$
n^{1 / 6}\left(\int_{0}^{1} A_{n}(t)^{p} \mathrm{~d} \mu(t)-m\right) \stackrel{d}{\rightarrow} N\left(0, \sigma^{2}\right)
$$

where

$$
m=\mathbb{E}\left[\zeta(0)^{p}\right] \int_{0}^{1} \frac{2^{p / 3} L^{\prime}(t)^{2 p / 3}}{\left|\lambda^{\prime}(t)\right|^{p / 3}} \mathrm{~d} \mu(t)
$$

and

$$
\sigma^{2}=\int_{0}^{1} \frac{2^{(2 p+5) / 3} L^{\prime}(t)^{(4 p+1) / 3}}{\left|\lambda^{\prime}(t)\right|^{(2 p+2) / 3}} w^{2}(t) \mathrm{d} t \int_{0}^{\infty} \operatorname{cov}\left(\zeta(0)^{p}, \zeta(s)^{p}\right) \mathrm{d} s .
$$

For the special cases that $\lambda$ is a probability density or a regression function, we recover Theorem 2.1 in Kulikov and Lopuhaä (2008) and Theorem 5.2 inDurot and Tocquet (2003), respectively. In order to prove Theorem 4 we first need some preliminary results. We aim at approximating the $L_{p}$-norm of $A_{n}$ by that of the Brownian motion version $A_{n}^{W}$ and then finding the asymptotic distribution for the latter one. To this end, we first need to relate the moments of $A_{n}$ to those of $A_{n}^{W}$. We start by showing that, for $J=E, W$, a rescaled version of $\Lambda_{n}^{J}$ can be approximated by the same process $Y_{n t}$ plus a linear term. This result corresponds to Lemma 4.1 in Kulikov and Lopuhaä (2008).

Lemma 5. Suppose that assumptions (A1)-(A2) are satisfied. Then, for $t \in(0,1)$ fixed, for $J=E, W$, and $s \in\left[-t n^{1 / 3},(1-t) n^{1 / 3}\right]$, it holds $n^{2 / 3} \Lambda_{n}^{J}\left(t+n^{-1 / 3} s\right)=Y_{n t}(s)+L_{n t}^{J}(s)+R_{n t}^{J}(s)$, where $L_{n t}^{J}(s)$ is linear in $s$ and $Y_{n t}(s)=n^{1 / 6}\left\{W_{n}\left(L\left(t+n^{-1 / 3} s\right)\right)-W_{n}(L(t))\right\}+\frac{1}{2} \lambda^{\prime}(t) s^{2}$. Moreover, for all $p \geq 1$,

$$
\mathbb{E}\left[\sup _{|s| \leq \log n}\left|R_{n t}^{W}(s)\right|^{p}\right]=O\left(n^{-p / 3}(\log n)^{3 p}\right),
$$

uniformly in $t \in(0,1)$. If, in addition $1 \leq p<q$ (with $q$ as in assumption (A2)), then

$$
\mathbb{E}\left[\sup _{|s| \leq \log n}\left|R_{n t}^{E}(s)\right|^{p}\right]=O\left(n^{-p / 3+p / q}\right)
$$

uniformly in $t \in(0,1)$.

Since the map $D_{I}$ is invariant under addition of linear terms, Lemma 5 allows us to approximate the moments of $A_{n}^{J}(t)=n^{2 / 3} D_{[0,1]} \Lambda_{n}^{J}$ by those of $\left[D_{H_{n t}} Y_{n t}\right](0)$ for some interval $H_{n t}$, as in Lemma 4.2 in Kulikov and Lopuhaä (2008).

Lemma 6. Suppose that assumptions (A1)-(A2) are satisfied. and let $Y_{n t}$ be the process defined in Lemma可. Define $H_{n t}=\left[-n^{1 / 3} t, n^{1 / 3}(1-t)\right] \cap[-\log n, \log n]$. Then for all $p \geq 1$, it holds

$$
\mathbb{E}\left[A_{n}^{W}(t)^{p}\right]=\mathbb{E}\left[\left[\mathrm{D}_{H_{n t}} Y_{n t}\right](0)^{p}\right]+o\left(n^{-1 / 6}\right),
$$


uniformly for $t \in(0,1)$. If, in addition $1 \leq p<\min (q, 2 q-7)$, with $q$ from condition (A2), then also

$$
\mathbb{E}\left[A_{n}^{E}(t)^{p}\right]=\mathbb{E}\left[\left[\mathrm{D}_{H_{n t}} Y_{n t}\right](0)^{p}\right]+o\left(n^{-1 / 6}\right),
$$

uniformly for $t \in(0,1)$.

The process $Y_{n t}$ has the same distribution as

$$
\tilde{Y}_{n t}=W\left(n^{1 / 3}\left(L\left(t+n^{-1 / 3} s\right)-L(t)\right)\right)+\frac{1}{2} \lambda^{\prime}(t) s^{2},
$$

which is close to the process $Z_{t}$ in (5) by continuity of Brownian motion. Lemma 4.3 in Kulikov and Lopuhaä (2008) is then used to show that the concave majorants at zero are sufficiently close. Note that, with by Brownian scaling, the process $c_{1}(t) Z_{t}\left(c_{2}(t) s\right)$ has the same distribution as the process $Z(s)$. As a consequence of Lemma 6 the moments of $A_{n}^{J}(t)$ can be related to those of the process $\zeta$. This formulated in the next lemma, which corresponds to Lemma 4.4 in Kulikov and Lopuhaä (2008).

Lemma 7. Suppose that assumptions (A1)-(A2) are satisfied. Then, for all $p \geq 1$,

$$
\mathbb{E}\left[A_{n}^{W}(t)^{p}\right]=\left(\frac{2 L^{\prime}(t)^{2}}{\left|\lambda^{\prime}(t)\right|}\right)^{p / 3} \mathbb{E}\left[\zeta(0)^{p}\right]+o\left(n^{-1 / 6}\right)
$$

uniformly in $t \in\left(n^{-1 / 3} \log n, 1-n^{-1 / 3} \log n\right)$ and

$$
\mathbb{E}\left[A_{n}^{W}(t)^{p}\right] \leq\left(\frac{2 L^{\prime}(t)^{2}}{\left|\lambda^{\prime}(t)\right|}\right)^{p / 3} \mathbb{E}\left[\zeta(0)^{p}\right]+o\left(n^{-1 / 6}\right)
$$

uniformly in $t \in(0,1)$. If, in addition $1 \leq p<\min (q, 2 q-7)$, where $q$ is from assumption (A2), then the same (in)equalities hold for $A_{N}^{E}(t)$.

In Lemmas 6 and 7 the moments of $A_{n}^{E}$ and $A_{n}^{W}$ are approximated by the moments of the same process. This suggests that the difference between them is of smaller order than $n^{-1 / 6}$. Indeed, on the events $N_{n t}^{J}$, where $A_{n}^{J}=n^{2 / 3} D_{I_{n t}} \Lambda_{n}^{J}$, we make use of Lemma 6 and the fact that $D_{I}$ is invariant under addition of linear functions to obtain that

$$
\sup _{t \in(0,1)}\left|n^{2 p / 3}\left[D_{I_{n t}} \Lambda_{n}^{E}\right](t)-n^{2 p / 3}\left[D_{I_{n t}} \Lambda_{n}^{W}\right](t)\right| \leq \sup _{t \in(0,1)} \sup _{|s| \leq \log n}\left\{\left|R_{n t}^{E}(s)\right|+\left|R_{n t}^{W}(s)\right|\right\},
$$

where the processes $R_{n t}^{J}$ converge to zero sufficiently fast. On the other hand, on $\left(N_{n t}^{J}\right)^{c}$ we just need the boundedness of the moments of $A_{n}^{J}$, which follows by Lemma 7 and the fact that the probability of these events is very small (Lemma 3).

Lemma 8. Suppose that assumptions (A1)-(A2) are satisfied. Then, for $1 \leq p<\min (q, 2 q-7)$, with $q$ from assumption (A2), it holds

$$
\begin{aligned}
\mathbb{E}\left[\left|A_{n}^{E}(t)^{p}-A_{n}^{W}(t)^{p}\right|\right] & =o\left(n^{-1 / 6}\right) \\
\mathbb{E}\left[\left|A_{n}^{E}(t)-A_{n}^{W}(t)\right|^{p}\right] & =o\left(n^{-1 / 6}\right)
\end{aligned}
$$

uniformly in $t \in(0,1)$. 
From Lemma 7 it follows that $n^{1 / 6}\left|m-\int_{0}^{1} \mathbb{E}\left[A_{n}^{W}(t)^{p}\right] \mathrm{d} t\right| \rightarrow 0$, where $m$ is the asymptotic mean in Theorem 4. Moreover, Lemma 8 implies that

$$
n^{1 / 6}\left|\int_{0}^{1} A_{n}^{E}(t)^{p} \mathrm{~d} t-\int_{0}^{1} A_{n}^{W}(t)^{p} \mathrm{~d} t\right| \leq n^{1 / 6} \int_{0}^{1}\left|A_{n}^{E}(t)^{p}-A_{n}^{W}(t)^{p}\right| \mathrm{d} t \rightarrow 0 .
$$

As a consequence, in order to prove Theorem 4, it suffices to prove asymptotic normality of its Brownian motion version

$$
T_{n}^{W}=n^{1 / 6} \int_{0}^{1}\left(A_{n}^{W}(t)^{p}-\mathbb{E}\left[A_{n}^{W}(t)^{p}\right]\right) \mathrm{d} \mu(t) .
$$

The proof of this is completely similar to that of Theorem 2.1 in Kulikov and Lopuhaä (2008). First, by using Theorem 1 for a Brownian version of $\zeta_{n t}$ and the mixing property of $A_{n}^{W}$ (this can be obtained in the same way as Lemma 4.6 in Kulikov and Lopuhaä (2008)), we derive the asymptotic variance of $T_{n}^{W}$ in the following lemma.

Lemma 9. Suppose that assumptions (A1)-(A3) are satisfied. Then, for every $p \geq 1$,

$$
\operatorname{Var}\left(n^{1 / 6} \int_{0}^{1} A_{n}^{W}(t)^{p} \mathrm{~d} \mu(t)\right) \rightarrow \int_{0}^{1} \frac{2^{(2 p+5) / 3} L^{\prime}(t)^{(4 p+1) / 3}}{\left|\lambda^{\prime}(t)\right|^{(2 p+2) / 3}} w^{2}(t) \mathrm{d} t \int_{0}^{\infty} \operatorname{cov}\left(\zeta(0)^{p}, \zeta(s)^{p}\right) \mathrm{d} s .
$$

The last step is proving the asymptotic normality of $T_{n}^{W}$. This is done by a big-blocks smallblocks argument, where the contribution of the small blocks to the asymptotic distribution is negligible, while the mixing property of $A_{n}^{W}$ allows us to approximate the sum over the big blocks by a sum of independent random variables which satisfy the assumptions of Lindeberg central limit theorem.

\section{Proofs}

Proof of Lemma Q 2. The proof is completely similar to that of Lemma $1.1 \mathrm{in} \mathrm{Kulikov}$ and Lopuhaä (2006), but this time $E_{n}=\sqrt{n}\left(\Lambda_{n}-\Lambda\right)$ and $\sup _{t \in[0,1]}\left|E_{n}(t)-B_{n} \circ L(t)\right|=O_{p}\left(n^{-1 / 2+1 / q}\right)$, according to (A2). Similar to the proof of Lemma 1.1 in Kulikov and Lopuhaä (2006), this means that

$$
X_{n t}(s)=n^{1 / 6}\left(W_{n}\left(L\left(t+s n^{-1 / 3}\right)\right)-W_{n}(L(t))\right)+O_{p}\left(n^{-1 / 3+1 / q}\right) \stackrel{d}{=} W\left(L^{\prime}(t) s\right)+R_{n}(s),
$$

where $\sup _{s \in I}\left|R_{n}(s)\right| \rightarrow 0$ in probability for compact $I \subset \mathbb{R}$. From here on the proof is the same as that of Lemma 1.1 in Kulikov and Lopuhaä (2006).

Proof Lemma 3, Let $\widehat{\lambda}_{n}^{W}$ be the left derivative of $\widehat{\Lambda}_{n}^{W}=\mathrm{CM}_{[0,1]} \Lambda_{n}^{W}$. Define the inverse process

$$
U_{n}^{W}(a)=\underset{t \in[0,1]}{\operatorname{argmax}}\left\{\Lambda_{n}^{W}(t)-a t\right\} \quad \text { and } \quad V_{n}^{W}(a)=n^{1 / 3}\left(L\left(U_{n}^{W}(a)\right)-L(g(a))\right),
$$


where $g$ denotes the inverse of $\lambda$. As in the proof of Lemma 1.3 in Kulikov and Lopuhaä (2006) [see (2.2)], we get

$$
\begin{aligned}
\mathbb{P}\left(\left(N_{n t}^{W}(d)\right)^{c}\right) \leq & \mathbb{P}\left(\widehat{\lambda}_{n}^{W}\left(t-n^{-1 / 3} d\right)=\widehat{\lambda}_{n}^{W}\left(t-n^{-1 / 3} d / 2\right)\right) \\
& +\mathbb{P}\left(\widehat{\lambda}_{n}^{W}\left(t+n^{-1 / 3} d\right)=\widehat{\lambda}_{n}^{W}\left(t+n^{-1 / 3} d / 2\right)\right) .
\end{aligned}
$$

Then, with $s=t-d n^{-1 / 3} / 2, x=d / 2$, and $\epsilon_{n}=\inf _{t \in[0,1]}\left|\lambda^{\prime}(t)\right| d n^{-1 / 3} / 8$, it holds (see (2.3) in Kulikov and Lopuhaä (2006)),

$$
\begin{aligned}
\mathbb{P}\left(\widehat{\lambda}_{n}^{W}\left(t-n^{-1 / 3} d\right)=\widehat{\lambda}_{n}^{W}\left(t-n^{-1 / 3} d / 2\right)\right) \leq & \mathbb{P}\left(\widehat{\lambda}_{n}^{W}\left(s+n^{-1 / 3} x\right)-\lambda\left(s+n^{-1 / 3} x\right)>\epsilon_{n}\right) \\
& +\mathbb{P}\left(\widehat{\lambda}_{n}^{W}(s)-\lambda(s)<-\epsilon_{n}\right) .
\end{aligned}
$$

Moreover, using the switching relation $\widehat{\lambda}_{n}^{W}(t) \leq a \Leftrightarrow U_{n}^{W}(a) \leq t$, we rewrite this probability as

$$
\begin{aligned}
& \mathbb{P}\left\{U_{n}^{W}\left(\lambda\left(s+n^{-1 / 3} x\right)+\epsilon_{n}\right)>s+n^{-1 / 3} x\right\} \\
& =\mathbb{P}\left\{V_{n}^{W}\left(\lambda\left(s+n^{-1 / 3} x\right)+\epsilon_{n}\right)>n^{1 / 3}\left(L\left(s+n^{-1 / 3} x\right)-L\left(g\left(\lambda\left(s+n^{-1 / 3} x\right)+\epsilon_{n}\right)\right)\right)\right\} \\
& =\mathbb{P}\left\{V_{n}^{W}\left(\lambda\left(s+n^{-1 / 3} x\right)+\epsilon_{n}\right)>\frac{\inf _{t \in[0,1]}\left|\lambda^{\prime}(t)\right| \inf _{t \in[0,1]} L^{\prime}(t) d}{8 \sup _{t \in[0,1]}\left|\lambda^{\prime}(t)\right|}\right\} .
\end{aligned}
$$

It suffices to show that there exists positive constants $C_{1}, C_{2}$ such that

$$
\mathbb{P}\left(V_{n}^{W}(a)>x\right) \leq C_{1} \mathrm{e}^{-C_{2} x^{3}}
$$

because then it follows that

$$
\mathbb{P}\left(V_{n}^{W}\left(\lambda\left(s+n^{-1 / 3} x\right)+\epsilon_{n}\right)>\frac{\inf _{t \in[0,1]}\left|\lambda^{\prime}(t)\right| \inf _{t \in[0,1]} L^{\prime}(t) d}{8 \sup _{t \in[0,1]}\left|\lambda^{\prime}(t)\right|}\right) \leq \tilde{C}_{1} \mathrm{e}^{-\tilde{C}_{2} d^{3}} .
$$

Similarly we can also bound the second probabilities in (9) and (10). Then the statement of the lemma follows immediately.

Now we prove (11). First write

$$
\begin{aligned}
V_{n}^{W}(a) & =n^{1 / 3}(L(\underset{t \in[0,1]}{\operatorname{argmax}}\{W(L(t))+\sqrt{n}(\Lambda(t)-a t)\})-L(g(a))) \\
& =n^{1 / 3}\left(\underset{s \in[L(0), L(1)]}{\operatorname{argmax}}\left\{W(s)+\sqrt{n}\left(\Lambda\left(L^{-1}(s)\right)-a L^{-1}(s)\right)\right\}-L(g(a))\right) .
\end{aligned}
$$

Using properties of the argmax functional we obtain that the right hand side is equal to the argmax of the process

$$
\begin{aligned}
& n^{1 / 6}\left\{W\left(n^{-1 / 3} s+L(g(a))\right)-W(L(g(a)))\right\} \\
& +n^{2 / 3}\left\{\Lambda\left(L^{-1}\left(n^{-1 / 3} s+L(g(a))\right)\right)-\Lambda(g(a))-a L^{-1}\left(n^{-1 / 3} s+L(g(a))\right)+a g(a)\right\}
\end{aligned}
$$

for $s \in I_{n}(a)=\left[n^{1 / 3}(L(0)-L(g(a))), n^{1 / 3}(L(1)-L(g(a)))\right]$. By Brownian motion scaling, $V_{n}^{W}(a)$ is equal in distribution to $\operatorname{argmax}_{t \in I_{n}(a)}\left\{W(t)-D_{a, n}(t)\right\}$, where $W$ is a standard two-sided Brownian 
motion originating from zero and

$$
\begin{aligned}
D_{a, n}(s)=-n^{2 / 3}\{\Lambda & \left(L^{-1}\left(n^{-1 / 3} s+L(g(a))\right)\right)-\Lambda(g(a)) \\
& \left.-a L^{-1}\left(n^{-1 / 3} s+L(g(a))\right)+a g(a)\right\} .
\end{aligned}
$$

By Taylor's formula and the assumptions on $\lambda$ and $L$, one can show that there exist a constant $c_{0}>$ 0 , independent of $n, a$ and $t$, such that $D_{a, n}(t) \geq c_{0} t^{2}$. Then (11) follows from Theorem 4 in Durot (2002), which proves the first statement.

To continue with the second statement, let $\widehat{\lambda}_{n}$ be the left derivative of $\widehat{\Lambda}_{n}$ and define the inverse process

$$
U_{n}(a)=\underset{t \in[0,1]}{\operatorname{argmax}}\left\{\Lambda_{n}(t)-a t\right\}, \quad \text { and } \quad V_{n}(a)=n^{1 / 3}\left(U_{n}(a)-g(a)\right),
$$

where $g$ denotes the inverse of $\lambda$. As in (9), we get

$$
\begin{aligned}
\mathbb{P}\left(\left(N_{n t}^{E}(d)\right)^{c}\right) \leq & \mathbb{P}\left(\widehat{\lambda}_{n}\left(t-n^{-1 / 3} d\right)=\widehat{\lambda}_{n}\left(t-n^{-1 / 3} d / 2\right)\right) \\
& +\mathbb{P}\left(\widehat{\lambda}_{n}\left(t+n^{-1 / 3} d\right)=\widehat{\lambda}_{n}\left(t+n^{-1 / 3} d / 2\right)\right) .
\end{aligned}
$$

where similar to (10),

$$
\begin{aligned}
\mathbb{P}\left(\widehat{\lambda}_{n}\left(t-n^{-1 / 3} d\right)=\widehat{\lambda}_{n}\left(t-n^{-1 / 3} d / 2\right)\right) \leq & \mathbb{P}\left(\widehat{\lambda}_{n}\left(s+n^{-1 / 3} x\right)-\lambda\left(s+n^{-1 / 3} x\right)>\epsilon_{n}\right) \\
& +\mathbb{P}\left(\widehat{\lambda}_{n}(s)-\lambda(s)<-\epsilon_{n}\right) .
\end{aligned}
$$

Then using the switching relation $\widehat{\lambda}_{n}(t) \leq a \Leftrightarrow U_{n}(a) \leq t$, we rewrite the first probability in (13) as

$$
\mathbb{P}\left(V_{n}\left(\lambda\left(s+n^{-1 / 3} x\right)+\epsilon_{n}\right)>\frac{\inf _{t \in[0,1]}\left|\lambda^{\prime}(t)\right| d}{8 \sup _{t \in[0,1]}\left|\lambda^{\prime}(t)\right|}\right) .
$$

According to Lemma 6.4 in Durot et al. (2012), there exists positive constants $C_{1}, C_{2}>0$, independent of $n, a$, and $x$, such that

$$
\mathbb{P}\left(V_{n}(a)>x\right) \leq \frac{C_{1} n^{1-q / 3}}{x^{2 q}}+2 \mathrm{e}^{-C_{2} x^{3}} .
$$

It follows that

$$
\mathbb{P}\left(V_{n}\left(\lambda\left(s+n^{-1 / 3} x\right)+\epsilon_{n}\right)>\frac{\inf _{t \in[0,1]}\left|\lambda^{\prime}(t)\right| d}{8 \sup _{t \in[0,1]}\left|\lambda^{\prime}(t)\right|}\right) \leq \frac{\tilde{C}_{1} n^{1-q / 3}}{d^{2 q}}+2 \mathrm{e}^{-\tilde{C}_{2} d^{3}} .
$$

Similarly we can also bound the second probabilities in (12) and (13). Then the statement of the lemma follows immediately.

Proof Theorem 11. The proof is similar to the proof of Theorem 1.1 in Kulikov and Lopuhaä (2006). We briefly sketch the main steps. Arguing as in the proof of Theorem 1.1 in Kulikov and Lopuhaä (2006), it suffices to show that for any compact $K \subset \mathbb{R}$, the process $\left\{A_{n}\left(t+s n^{-1 / 3}\right): s \in K\right\}$ converges in distribution to the process $\left\{\left[D_{\mathbb{R}} Z_{t}\right](s): s \in K\right\}$ on $D(K)$, the space of cadlag 
functions on $K$, where $Z_{t}$ is defined in (5). By definition $A_{n}\left(t+s n^{-1 / 3}\right)=\left[D_{I_{n t}} E_{n t}\right](s)$, for $s \in I_{n t}=\left[-t n^{1 / 3},(1-t) n^{1 / 3}\right]$, where $E_{n t}$ is defined in (5). To prove convergence in distribution, we show that for any bounded continuous function $g: D(K) \rightarrow \mathbb{R}$,

$$
\left|\mathbb{E}\left[g\left(D_{I_{n t}} E_{n t}\right)\right]-\mathbb{E}\left[g\left(D_{\mathbb{R}} Z_{t}\right)\right]\right| \rightarrow 0
$$

To this end, we choose $d>0$ sufficiently large, such that $K \subset[-d / 2, d / 2] \subset[-d, d]=I$ and take $n$ sufficiently large so that $I \subset I_{n t}$. Then, similar to inequality (2.7) in Kulikov and Lopuhaä (2006), the triangular inequality yields

$$
\begin{aligned}
\left|\mathbb{E}\left[g\left(D_{I_{n t}} E_{n t}\right)\right]-\mathbb{E}\left[D_{\mathbb{R}} Z_{t}\right]\right| \leq & \left|\mathbb{E}\left[g\left(D_{I_{n t}} E_{n t}\right)\right]-\mathbb{E}\left[D_{I} E_{n t}\right]\right| \\
& +\left|\mathbb{E}\left[g\left(D_{I} E_{n t}\right)\right]-\mathbb{E}\left[D_{I} Z_{t}\right]\right|+\left|\mathbb{E}\left[g\left(D_{I} Z_{t}\right)\right]-\mathbb{E}\left[D_{\mathbb{R}} Z_{t}\right]\right|
\end{aligned}
$$

In the same way as in Kulikov and Lopuhaä (2006), the three terms on the right hand side are shown to go to zero. For the last term on the right hand side of (15), the argument is exactly the same and makes use of their Lemma 1.2. The first term on the right hand side of (15) is bounded similar to their inequality (2.9) and then uses Lemma 3. For the second term on the right hand side of (15), note that from Lemma 2, it follows that

$$
Z_{n t}(s)=n^{2 / 3}\left(\Lambda_{n}\left(t+s n^{-1 / 3}\right)-\Lambda_{n}(t)-\left(\Lambda\left(t+s n^{-1 / 3}\right)-\Lambda(t)\right)\right)+\frac{1}{2} \lambda^{\prime}(t) s^{2},
$$

converges in distribution to $Z_{t}$. Therefore, because of the continuity of the mapping $D_{I}$, we get $\left|\mathbb{E}\left[h\left(D_{I} Z_{n t}\right)\right]-\mathbb{E}\left[h\left(D_{I} Z_{t}\right)\right]\right| \rightarrow 0$, for any $h: D(I) \rightarrow \mathbb{R}$ bounded and continuous. Moreover, we now have $E_{n t}(s)=Z_{n t}(s)+n^{2 / 3} \Lambda_{n}(t)+\lambda(t) s n^{1 / 3}+R_{n t}(s)$, where

$$
R_{n t}(s)=n^{2 / 3}\left(\Lambda\left(t+s n^{-1 / 3}\right)-\Lambda(t)-\lambda(t) s n^{-1 / 3}-\frac{1}{2} \lambda^{\prime}(t) s^{2} n^{-2 / 3}\right) .
$$

Similar to the argument leading up to (2.11) in Kulikov and Lopuhaä (2006), from the continuity of $D_{I}$, its invariance under addition of linear functions, and continuity of $\lambda^{\prime}$, it follows that $\left|\mathbb{E}\left[g\left(D_{I} Z_{n t}\right)\right]-\mathbb{E}\left[g\left(D_{I} E_{n t}\right)\right]\right| \rightarrow 0$. This establishes (14) and finishes the proof.

Proof of Lemma 5. By a Taylor expansion, together with (6), we can write

$$
n^{2 / 3} \Lambda_{n}^{W}\left(t+n^{-1 / 3} s\right)=Y_{n t}(s)+L_{n t}^{W}(s)+R_{n t}^{W}(s),
$$

where $L_{n t}^{W}(s)=n^{2 / 3} \Lambda(t)+n^{1 / 6} W_{n}(L(t))+n^{1 / 3} \lambda(t) s$ and

$$
R_{n t}^{W}(s)=n^{2 / 3}\left(\Lambda\left(t+n^{-1 / 3} s\right)-\Lambda(t)-n^{-1 / 3} \lambda(t) s-\frac{1}{2} n^{-2 / 3} \lambda^{\prime}(t) s^{2}\right)=\frac{1}{6} n^{-1 / 3} \lambda^{\prime \prime}\left(\theta_{1}\right) s^{3}
$$

for some $\left|\theta_{1}-t\right| \leq n^{-1 / 3}|s|$. Then, from the assumptions (A1)-(A2), it follows that

$$
\sup _{|s| \leq \log n}\left|R_{n t}^{W}(s)\right|^{p}=O\left(n^{-p / 3}(\log n)^{3 p}\right)
$$


uniformly in $t \in(0,1)$. Similarly, we also obtain

$$
\begin{aligned}
n^{2 / 3} \Lambda_{n}^{E}\left(t+n^{-1 / 3} s\right)= & n^{2 / 3} \Lambda_{n}^{W}\left(t+n^{-1 / 3} s\right)+n^{1 / 6}\left(E_{n}\left(t+n^{-1 / 3} s\right)-B_{n}\left(L\left(t+n^{-1 / 3} s\right)\right)\right) \\
& -n^{1 / 6} \zeta_{n}\left(L(t)+L^{\prime}(t) n^{-1 / 3} s\right) \\
& \quad-n^{1 / 6} \zeta_{n}\left(L\left(t+n^{-1 / 3} s\right)-L(t)-L^{\prime}(t) n^{-1 / 3} s\right) \\
= & Y_{n t}(s)+L_{n t}^{E}(s)+R_{n t}^{E}(s),
\end{aligned}
$$

where $L_{n t}^{E}(s)=L_{n t}^{W}(s)-n^{1 / 6} \zeta_{n} L(t)-n^{-1 / 6} \zeta_{n} L^{\prime}(t) s$ and

$$
R_{n t}^{E}(s)=R_{n t}^{W}(s)+n^{1 / 6}\left(E_{n}\left(t+n^{-1 / 3} s\right)-B_{n}\left(L\left(t+n^{-1 / 3} s\right)\right)\right)-\frac{1}{2} n^{-1 / 2} \zeta_{n} L^{\prime \prime}\left(\theta_{2}\right) s^{2},
$$

for some $\left|\theta_{2}-t\right| \leq n^{-1 / 3}|s|$. Let $S_{n}=\sup _{s \in[0,1]}\left|E_{n}(s)-B_{n}(L(s))\right|$. From assumption (A2) we have $\mathbb{P}\left(S_{n}>n^{-1 / 2+1 / q} x\right) \leq C_{q} x^{-q}$ and it follows that

$$
\begin{aligned}
\mathbb{E}\left[S_{n}^{p}\right] & =\int_{0}^{\infty} \mathbb{P}\left(S_{n}^{p} \geq x\right) \mathrm{d} x=p \int_{0}^{\infty} y^{p-1} \mathbb{P}\left(S_{n} \geq y\right) \mathrm{d} y \\
& =p n^{-p / 2+p / q} \int_{0}^{\infty} x^{p-1} \mathbb{P}\left(S_{n} \geq n^{-1 / 2+1 / q} x\right) \mathrm{d} x \\
& \leq p n^{-p / 2+p / q}\left\{\int_{0}^{1} x^{p-1} \mathrm{~d} x+C_{q} \int_{1}^{\infty} x^{p-1-q} \mathrm{~d} x\right\}=O\left(n^{-p / 2+p / q}\right),
\end{aligned}
$$

if $p<q$. Consequently $\mathbb{E}\left[\sup _{|s| \leq \log n}\left|R_{n t}^{E}(s)\right|^{p}\right]=O\left(n^{-p / 3+p / q}\right)$.

Proof of Lemma [6. Note that we can write $A_{n}^{J}(t) \mathbb{1}_{N_{n t}^{J}}=n^{2 / 3}\left[\mathrm{D}_{I_{n t}} \Lambda_{n}^{J}\right](t) \mathbb{1}_{N_{n t}^{J}}$. We have

$$
\mathbb{E}\left[A_{n}^{J}(t)^{p}\right]=n^{2 p / 3} \mathbb{E}\left[\left[\mathrm{D}_{I_{n t}} \Lambda_{n}^{J}\right](t)^{p}\right]+\mathbb{E}\left[\left(A_{n}^{J}(t)^{p}-n^{2 p / 3}\left[\mathrm{D}_{I_{n t}} \Lambda_{n}^{J}\right](t)^{p}\right) \mathbb{1}_{\left(N_{n t}^{J}\right)^{c}}\right] .
$$

To bound the second term on the right hand side, first note that

$$
\left|A_{n}^{J}(t)^{p}-n^{2 p / 3}\left[\mathrm{D}_{I_{n t}} \Lambda_{n}^{J}\right](t)^{p}\right| \leq 2 A_{n}^{J}(t)^{p},
$$

because the LCM on $[0,1]$ always lies above the LCM over $I_{n t}$. Since $\Lambda$ is concave, we have that

$$
\begin{aligned}
\left|\mathrm{CM}_{[0,1]} \Lambda_{n}^{E}-\Lambda_{n}^{E}\right| & \leq \mid \mathrm{CM}_{[0,1]} \Lambda_{n}^{E}-\left[\mathrm{CM}_{[0,1]} \Lambda|+| \Lambda_{n}^{E}-\Lambda|+| \mathrm{CM}_{[0,1]} \Lambda-\Lambda \mid\right. \\
& =\mid \mathrm{CM}_{[0,1]} \Lambda_{n}^{E}-\left[\mathrm{CM}_{[0,1]} \Lambda|+| \Lambda_{n}^{E}-\Lambda\left|\leq 2 \sup _{s \in[0,1]}\right| \Lambda_{n}^{E}(s)-\Lambda(s) \mid\right.
\end{aligned}
$$

which means that $0 \leq A_{n}^{E}(t)^{p} \leq 2^{p} n^{2 p / 3} \sup _{s \in[0,1]}\left|\Lambda_{n}^{E}(s)-\Lambda(s)\right|^{p}$. Furthermore,

$$
0 \leq A_{n}^{W}(t)^{p} \leq 2^{p} n^{2 p / 3}\left\{\Lambda(1)+n^{-1 / 2} \sup _{s \in[0,1]}\left|W_{n}(s)\right|\right\}^{p} .
$$

In contrast to Kulikov and Lopuhaä (2008) it is more convenient to treat both cases separately. For the case $J=E$, with (17), we find that

$$
\mathbb{E}\left[\left(A_{n}^{E}(t)^{p}-n^{2 p / 3}\left[\mathrm{D}_{I_{n t}} \Lambda_{n}^{E}\right](t)^{p}\right) \mathbb{1}_{\left(N_{n t}^{E}\right)^{c}}\right] \leq 2^{p+1} n^{2 p / 3} \mathbb{E}\left[\sup _{s \in[0,1]}\left|\Lambda_{n}^{E}(s)-\Lambda(s)\right|^{p} \mathbb{1}_{\left(N_{n t}^{J}\right)^{c}}\right],
$$


where

$$
\sup _{s \in[0,1]}\left|\Lambda_{n}^{E}(s)-\Lambda(s)\right|^{p} \leq 2^{p}\left\{\sup _{s \in[0,1]}\left|\Lambda_{n}^{E}(s)-\Lambda(s)-n^{-1 / 2} W_{n}(L(s))\right|^{p}+n^{-p / 2} \sup _{s \in[0,1]}\left|W_{n}(L(s))\right|^{p}\right\} .
$$

For the first term on the right hand side we get with Hölder's inequality

$$
\begin{aligned}
& n^{2 p / 3} \mathbb{E}\left[\sup _{s \in[0,1]}\left|\Lambda_{n}^{E}(s)-\Lambda(s)-n^{-1 / 2} W_{n}(L(s))\right|^{p} \mathbb{1}_{\left(N_{n t}^{E}\right)^{c}}\right] \\
& \leq n^{2 p / 3} \mathbb{E}\left[\sup _{s \in[0,1]}\left|\Lambda_{n}^{E}(s)-\Lambda(s)-n^{-1 / 2} W_{n}(L(s))\right|^{p \ell}\right]^{1 / \ell} \mathbb{P}\left(\left(N_{n t}^{E}\right)^{c}\right)^{1 / \ell^{\prime}} \\
& =n^{2 p / 3} O\left(n^{-p+p / q}\right) O\left(n^{1-q / 3}(\log n)^{-2 q}+\mathrm{e}^{-C(\log n)^{3}}\right)^{1 / \ell^{\prime}},
\end{aligned}
$$

for any $\ell, \ell^{\prime}>1$ such that $1 / \ell+1 / \ell^{\prime}=1$, according to (16) and Lemma 3. When $q>6$, then the right hand side is of the order $o\left(n^{-1 / 6}\right)$. For the second term, with Hölder's inequality

$$
n^{2 p / 3} \mathbb{E}\left[\sup _{s \in[0,1]}\left|W_{n}(L(s))\right|^{p} \mathbb{1}_{\left(N_{n t}^{E}\right)^{c}}\right] \leq n^{2 p / 3} \mathbb{E}\left[\sup _{s \in[0, L(1)]}\left|W_{n}(s)\right|^{p \ell}\right]^{1 / \ell} \mathbb{P}\left(\left(N_{n t}^{E}\right)^{c}\right)^{1 / \ell^{\prime}}
$$

for any $\ell, \ell^{\prime}>1$ such that $1 / \ell+1 / \ell^{\prime}=1$. Since all moments of $\sup _{s \in[0, L(1)]}\left|W_{n}(s)\right|$ are finite, it follows from Lemma 3 that the right hand side is of the order

$$
n^{2 p / 3} \mathbb{E}\left[\sup _{s \in[0,1]}\left|W_{n}(L(s))\right|^{p} \mathbb{1}_{\left(N_{n t}^{E}\right)^{c}}\right] \leq n^{2 p / 3} O\left(n^{1-q / 3}(\log n)^{-2 q}+\mathrm{e}^{-C(\log n)^{3}}\right)^{1 / \ell^{\prime}} .
$$

Hence, because $q>6$ and $p<2 q-7$, it follows that $\left|A_{n}^{E}(t)^{p}-n^{2 p / 3}\left[\mathrm{D}_{I_{n t}} \Lambda_{n}^{E}\right](t)\right|=o\left(n^{-1 / 6}\right)$.

Next, consider the case $J=W$. Then with (17) and Cauchy-Schwarz, we find

$$
\begin{aligned}
& \mathbb{E}\left[\left(A_{n}^{W}(t)^{p}-n^{2 p / 3}\left[\mathrm{D}_{I_{n t}} \Lambda_{n}^{W}\right](t)^{p}\right) \mathbb{1}_{\left(N_{n t}^{W}\right)^{c}}\right] \\
& \leq 2^{p+1} n^{2 p / 3}\left\{\mathbb{E}\left[\left(\Lambda(1)+n^{-1 / 2} \sup _{s \in[0,1]}\left|W_{n}(s)\right|\right)^{2 p}\right]\right\}^{1 / 2}\left\{\mathbb{P}\left(\left(N_{n t}^{W}\right)^{c}\right)\right\}^{1 / 2} .
\end{aligned}
$$

Again using that all moments of $\sup _{s \in[0, L(1)]}\left|W_{n}(s)\right|$ are finite, according to Lemma 3, the right hand side is of the order $n^{2 p / 3} O\left(\mathrm{e}^{-C(\log n)^{3}}\right)=o\left(n^{-1 / 6}\right)$. It follows that for $J=E, W$,

$$
\mathbb{E}\left[A_{n}^{J}(t)^{p}\right]=n^{2 p / 3} \mathbb{E}\left[\left[\mathrm{D}_{I_{n t}} \Lambda_{n}^{J}\right](t)^{p}\right]+o\left(n^{-1 / 6}\right) .
$$

Moreover, Lemma 5 implies that $n^{2 / 3}\left[\mathrm{D}_{I_{n t}} \Lambda_{n}^{J}\right](t)=\left[\mathrm{D}_{H_{n t}} Y_{n t}\right](0)+\Delta_{n t}$, where $\Delta_{n t}=\left[\mathrm{D}_{H_{n t}}\left(Y_{n t}+\right.\right.$ $\left.\left.R_{n t}^{J}\right)\right](0)-\left[\mathrm{D}_{H_{n t}} Y_{n t}\right](0)$. From Lemma 5, we have

$$
\mathbb{E}\left|\Delta_{n t}\right|^{p} \leq 2^{p} \mathbb{E}\left[\sup _{|s| \leq \log n}\left|R_{n t}^{J}(s)\right|^{p}\right]=O\left(n^{-p / 3+p / q}\right) .
$$

Then as in Lemma 4.2 in Kulikov and Lopuhaä (2008), one can show that

$$
\begin{aligned}
\mathbb{E}\left[A_{n}^{J}(t)^{p}\right] & =\mathbb{E}\left[\left[\mathrm{D}_{H_{n t}} Y_{n t}(0)^{p}\right]+\epsilon_{n t}+o\left(n^{-1 / 6}\right)\right. \\
& =\mathbb{E}\left[\left[\mathrm{D}_{H_{n t}} Y_{n t}(0)^{p}\right]+O\left(n^{-1 / 3+1 / q}(\log n)^{2 p-2}\right)+o\left(n^{-1 / 6}\right)\right. \\
& =\mathbb{E}\left[\left[\mathrm{D}_{H_{n t}} Y_{n t}(0)^{p}\right]+o\left(n^{-1 / 6}\right) .\right.
\end{aligned}
$$

This finishes the proof. 
Proof of Lemma [7. The proof is exactly the same as the one for Lemma $4.4 \mathrm{in}$ Kulikov and Lopuhaä (2008). Define $J_{n t}=\left[n^{1 / 3}\left(L\left(a_{n t}\right)-L(t)\right) / L^{\prime}(t), n^{1 / 3}\left(L\left(b_{n t}\right)-L(t)\right) / L^{\prime}(t)\right]$, where $a_{n t}=\max (0, t-$ $\left.n^{-1 / 3} \log n\right)$ and $b_{n t}=\min \left(1, t+n^{-1 / 3} \log n\right)$. Furthermore, here we take

$$
\phi_{n t}(s)=\frac{n^{1 / 3}\left(L\left(t+n^{-1 / 3} s\right)-L(t)\right)}{L^{\prime}(t)} .
$$

As in the proof of Lemma 4.4 in Kulikov and Lopuhaä (2008), it follows that $1-\alpha_{n} \leq \phi_{n t}(s) / s \leq$ $1+\alpha_{n}$, for $s \in H_{n t}$, the interval from Lemma [6, and $\alpha_{n}=C_{1} n^{-1 / 3} \log n$, with $C_{1}>0$ only depending on $L^{\prime}$. Let $Z_{t}$ be the process in (5). Then

$$
\left(Z_{t} \circ \phi_{n t}\right)(s)=\tilde{Y}_{n t}+\frac{1}{2} \lambda^{\prime}(t) s^{2}\left(\frac{\phi_{n t}(s)^{2}}{s^{2}}-1\right),
$$

where $\tilde{Y}_{n t}$ is defined in (8). Lemma 4.3 in Kulikov and Lopuhaä (2008), then allows us to approximate the moments of $\left[D_{H_{n t}} \tilde{Y}_{n t}\right](0)$ by the moments of $\left[D_{J_{n t}} Z_{t}\right](0)$. Completely similar to the proof of Lemma 4.4 in Kulikov and Lopuhaä (2008), the result now follows from Lemma [6 and Brownian scaling.

Proof of Lemma 8. Let $I_{n t}$ and $N_{n t}^{J}$ be as in Lemma 6] and define $K_{n t}=N_{n t}^{E} \cap N_{n t}^{W}$. Then

$$
\begin{aligned}
\mathbb{E}\left|A_{n}^{E}(t)^{p}-A_{n}^{W}(t)^{p}\right|= & n^{2 p / 3} \mathbb{E}\left|\left[D_{I_{n t}} \Lambda_{n}^{E}\right](t)^{p}-\left[D_{I_{n t}} \Lambda_{n}^{W}\right](t)^{p}\right| \mathbb{1}_{K_{n t}} \\
& +\mathbb{E}\left|A_{n}^{E}(t)^{p}-A_{n}^{W}(t)^{p}\right| \mathbb{1}_{K_{n t}^{c}} .
\end{aligned}
$$

We bound the two terms on the right hand side, following the same line of reasoning as in Lemma 4.5 in Kulikov and Lopuhaä (2008). Using that according to Lemma 3 ,

$$
\mathbb{P}\left(K_{n t}^{c}\right) \leq \mathbb{P}\left(\left(N_{n t}^{E}\right)^{c}\right)+\mathbb{P}\left(\left(N_{n t}^{W}\right)^{c}\right)=O\left(n^{1-q / 3}(\log n)^{-2 q}+\mathrm{e}^{-C(\log n)^{3}}\right),
$$

the second term on the right hand side of (19) is of the order $O\left(\mathbb{P}\left(K_{n t}^{c}\right)^{1 / 2}\right)=o\left(n^{-1 / 6}\right)$, because $q>6$. On the other hand, the first term on the right hand side of (19) can be bounded by

$$
p\left\{\mathbb{E}\left[\left(A_{n}^{E}(t)^{p-1}+A_{n}^{W}(t)^{p-1}\right)^{2}\right]\right\}^{1 / 2}\left\{\mathbb{E}\left[\left(\sup _{|s| \leq \log n}\left|R_{n t}^{E}\right|+\sup _{|s| \leq \log n}\left|R_{n t}^{W}\right|\right)^{2}\right]\right\}^{1 / 2},
$$

where the right hand side is of the order $O\left(n^{-1 / 3+1 / q}\right)=o\left(n^{-1 / 6}\right)$, according to Lemmas 5 and 7

In the same way, we have $\mathbb{E}\left[\left|A_{n}^{E}(t)-A_{n}^{W}(t)\right|^{p} \mathbb{1}_{K_{n t}^{c}}\right]=O\left(\mathbb{P}\left(K_{n t}^{c}\right)^{1 / 2}\right)=o\left(n^{-1 / 6}\right)$ and

$$
n^{2 p / 3} \mathbb{E}\left[\left|\left[\mathrm{D}_{I_{n t}} \Lambda_{n}^{E}\right](t)-\left[\mathrm{D}_{I_{n t}} \Lambda_{n}^{W}\right](t)\right|^{p} \mathbb{1}_{K_{n t}}\right] \leq \mathbb{E}\left[\left(\sup _{|s| \leq \log n}\left|R_{n t}^{E}\right|+\sup _{|s| \leq \log n}\left|R_{n t}^{W}\right|\right)^{p}\right]
$$

which is of the order $O\left(n^{-p / 3+p / q}\right)=o\left(n^{-1 / 6}\right)$, according to Lemma 5 ,

Proof of Lemma 9. The proof is completely similar to the proof of Lemma 4.7 in Kulikov and Lopuhaä (2008). For $t \in(0,1)$ fixed, and $t+c_{2}(t) s n^{-1 / 3} \in(0,1)$, let $\zeta_{n t}(s)=c_{1}(t) A_{n}^{W}\left(t+c_{2}(t) s n^{-1 / 3}\right)$, 
where $A_{n}^{W}$ is defined in (6) and $c_{1}(t)$ and $c_{2}(t)$ are defined in (4). According to Theorem 1 , $\zeta_{n t}$ converges in distribution to $\zeta$, as defined in (2). As in the proof of Lemma 4.7 in Kulikov and Lopuhaä (2008), Lemmal7yields that, for $s, t$, and $k$ fixed, the sequence $\zeta_{n t}^{W}(s)^{k}$ is uniformly integrable, so that the moments of $\left(\zeta_{n t}^{W}(0)^{k}, \zeta_{n t}^{W}(s)^{k}\right)$ converge to the corresponding moments of $\left(\zeta(0)^{k}, \zeta(s)^{k}\right)$.

Furthermore, the process $\left\{A_{n}^{W}(t): t \in(0),\right\}$ is strong mixing, i.e., for $d>0$,

$$
\sup |P(A \cap B)-P(A) P(B)|=\alpha_{n}(d)=48 \mathrm{e}^{-C n d^{3}}
$$

where $C>0$ only depends on $\lambda$ and $L$ from (A2), and where the supremum is taken over all sets

$A \in \sigma\left\{A_{n}^{W}(s): 0 \leq s \leq t\right\}$ and $B \in \sigma\left\{A_{n}^{W}(s): t+d \leq s<1\right\}$. This can be obtained by arguing completely the same as in the proof of Lemma 4.6 in Kulikov and Lopuhaä (2008). The rest of the proof is the same as that of Lemma 4.7 in Kulikov and Lopuhaä (2008).

Proof of Theorem 因. The proof is completely similar to the proof of Theorem $2.1 \mathrm{in}$ Kulikov and Lopuhaä (2008), by using the method of big-blocks small-blocks and the exponential decreasing mixing function $\alpha_{n}$ from (20).

\section{References}

\section{References}

Brunk, H. D., 1958. On the estimation of parameters restricted by inequalities. Ann. Math. Statist. $29,437-454$.

Csörgö, M., Horváth, L., 1988. Central limit theorems for $L_{p}$-norms of density estimators. Probab. Theory Related Fields 80 (2), 269-291.

Durot, C., 2002. Sharp asymptotics for isotonic regression. Probab. Theory Related Fields 122 (2), $222-240$.

Durot, C., 2007. On the $\mathbb{L}_{p}$-error of monotonicity constrained estimators. Ann. Statist. 35 (3), 1080-1104.

Durot, C., Groeneboom, P., Lopuhaä, H. P., 2013. Testing equality of functions under monotonicity constraints. J. Nonparametr. Stat. 25 (4), 939-970.

Durot, C., Kulikov, V. N., Lopuhaä, H. P., 2012. The limit distribution of the $L_{\infty}$-error of Grenander-type estimators. Ann. Statist. 40 (3), 1578-1608.

Durot, C., Lopuhaä, H. P., 2014. A Kiefer-Wolfowitz type of result in a general setting, with an application to smooth monotone estimation. Electron. J. Stat. 8 (2), 2479-2513. 
Durot, C., Tocquet, A.-S., 2003. On the distance between the empirical process and its concave majorant in a monotone regression framework. Ann. Inst. H. Poincaré Probab. Statist. 39 (2), $217-240$.

Grenander, U., 1956. On the theory of mortality measurement. II. Skand. Aktuarietidskr. 39, 125-153 (1957).

Groeneboom, P., Jongbloed, G., 2013. Smooth and non-smooth estimates of a monotone hazard. In: From probability to statistics and back: high-dimensional models and processes. Vol. 9 of Inst. Math. Stat. (IMS) Collect. Inst. Math. Statist., Beachwood, OH, pp. 174-196.

Huang, J., Wellner, J. A., 1995. Estimation of a monotone density or monotone hazard under random censoring. Scand. J. Statist. 22 (1), 3-33.

Kiefer, J., Wolfowitz, J., 1976. Asymptotically minimax estimation of concave and convex distribution functions. Z. Wahrscheinlichkeitstheorie und Verw. Gebiete 34 (1), 73-85.

Kulikov, V. N., Lopuhaä, H. P., 2006. The limit process of the difference between the empirical distribution function and its concave majorant. Statist. Probab. Lett. 76 (16), 1781-1786.

Kulikov, V. N., Lopuhaä, H. P., 2008. Distribution of global measures of deviation between the empirical distribution function and its concave majorant. J. Theoret. Probab. 21 (2), 356-377.

Lopuhaä, H. P., Musta, E., 2017. Smooth estimation of a monotone hazard and a monotone density under random censoring. Statist. Neerlandica 71, 58-82.

Lopuhaä, H. P., Nane, G. F., 2013. Shape constrained non-parametric estimators of the baseline distribution in Cox proportional hazards model. Scand. J. Stat. 40 (3), 619-646.

Mammen, E., 1991. Estimating a smooth monotone regression function. Ann. Statist. 19 (2), $724-740$.

Sen, B., Banerjee, M., Woodroofe, M., 2010. Inconsistency of bootstrap: the Grenander estimator. Ann. Statist. 38 (4), 1953-1977.

Wang, X., Woodroofe, M., 2007. A Kiefer-Wolfowitz comparison theorem for Wicksell's problem. Ann. Statist. 35 (4), 1559-1575.

Wang, Y., 1994. The limit distribution of the concave majorant of an empirical distribution function. Statist. Probab. Lett. 20 (1), 81-84. 